\title{
$\boldsymbol{u}^{b}$
}

$b$ UNIVERSITÄT

BER N

Faculty of Business, Economics and Social Sciences

Department of Social Sciences

University of Bern Social Sciences Working Paper No. 31

\section{Color palettes for Stata graphics}

Ben Jann

April 12, 2018

http://ideas.repec.org/p/bss/wpaper/31.html

http://econpapers.repec.org/paper/bsswpaper/31.htm 


\title{
Color palettes for Stata graphics
}

\author{
Ben Jann \\ University of Bern
}

\begin{abstract}
This paper introduces a command called colorpalette that provides a wide variety of color palettes and color generators for use in Stata graphics. Supported are color palettes from official Stata's graph schemes, a selection of palettes that have been proposed by users, standard collections such as the ColorBrewer or D3.js palettes, as well as HSV and HCL color generators. As a byproduct, the paper also introduces commands for marker symbol and line pattern palettes.
\end{abstract}

Keywords: st0001, palettes, colorpalette, symbolpalette, linepalette, graph, graphics, color, color spaces

\section{Introduction}

Stata features a set of about 50 named colors that can be used in graphs (see $[\mathrm{G}]$ colorstyle). Given the diverse needs of users, a set of 50 predefined colors is rather limited. Alternative colors are supported, but have to be specified by their RGB, CMYK, or HSV values. To increase the number of easily accessible colors, the new colorpalette command provides a wide variety of predefined palettes and also features HSV (HueSaturation-Value) and HCL (Hue-Chroma-Luminance) color generators. Furthermore, it supports additional input formats for custom colors, such as hex triplets, and allows generating colors over a range if intensity or opacity levels.

A byproduct of colorpalette are two additional commands, symbolpalette and linepalette, that provide palettes of marker symbols and line pattern. These commands are briefly presented in the Appendix.

To install the new commands and view the documentation, type:

\footnotetext{
- scc install palettes, replace

- help palettes
}

\section{Syntax and basic usage}

The colorpalette command has two syntax variants. Syntax 1 is used to retrieve colors from one or multiple palettes. The colors are returned in $r()$ and, by default, displayed in a graph. The syntax is

colorpalette [argument] [, palette_options graph_options $]$

where argument is 


$$
\text { palette }[\text { [, palette_options }] /[\text { palette }[\text {, palette_options }] / \ldots]]
$$

and palette is a named palette as described below, or a space-separated list of named colors, RGB values, CMYK values, or HSV values according to [G] colorstyle, HCL values specified as "hcl $h c l$ ", where $h$ specifies the hue (dominant wavelength in degrees of the 360 degree color wheel), $c$ specifies the chroma (colorfulness; $c \geq 0$ ), and $l$ specifies the luminance (brightness, amount of gray; $l \in[0,100]$ ), or hex triplets specified as \# rrggbb, where $r r, g g$, and $b b$ are the two digit hex codes (or one digit abbreviations) for red, green, and blue. The specified colors can include intensity adjustment and, since Stata 15 , an opacity level specified as "color $[* i n t][\% o p]$ ", where int $\in[0,1)$ makes the color lighter, int $>1$ makes the color darker, and op is a number between 0 (fully transparent) and 100 (fully opaque).

Syntax 2 is used to display an overview of multiple palettes in a single graph, without returning the colors in $r()$. The syntax is

colorpalette $[$, palette_options graph_options $]:$ pspec $[/$ pspec / ...

where pspec is

$$
\text { palette [, palette_options] }
$$

or . to insert a gap.

\section{Palette options}

$\mathrm{n}$ (\#) specifies the size of the palette (the number of colors). In many cases this just selects the first \# colors from the palette and is thus equivalent to select(1/\#). However, some color schemes return colors that adjust to the size of the palette.

select (numlist) selects and orders the colors retrieved from the palette.

reverse returns the palette in reverse order.

intensity (numlist) applies color intensity adjustment. Values between 0 and 1 make the colors lighter; values larger than one make the colors darker. Specify multiple values to use different adjustments across the selected colors. The list of adjustments will be recycled if it is shorter than the list of selected colors. Likewise, colors will be recycled if the list of adjustments is longer than the palette.

opacity (numlist) sets the opacity level(s) (requires Stata 15). Values must be between 0 (fully transparent) and 100 (fully opaque). Specify multiple values to use different opacity levels across the selected colors. The list of opacity levels will be recycled if it is shorter than the list of selected colors. Likewise, colors will be recycled if the list of opacity levels is longer than the palette.

The above options are supported by all palettes, but some palettes also have additional options; see the descriptions of the palettes below. 


\section{Common graph options}

title(string) specifies a custom title for the graph.

gropts (twoway_options) provides options to be passed through to the graph command; see $[\mathrm{G}]$ twoway_options.

\section{Additional graph options for syntax 1}

nograph suppresses the graph.

rows (\#) specifies the minimum number of rows in the graph. The default is 5 .

\section{Additional graph options for syntax 2}

horizontal displays the palettes horizontally. This is the default.

vertical displays the palettes vertically.

plabels (strlist) provides custom labels for the palettes. Enclose labels with spaces in double quotes.

lcolor (colorstyle) specifies a custom outline color. The default is to use the same color as for the fill.

lwidth (linewidthstyle) specifies a custom outline thickness. The default is lwidth(vthin).

\section{Stored results}

Under syntax 1, colorpalette stores the following in $r()$ :

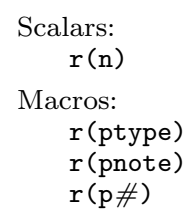

$r(n) \quad$ number of colors

$$
\begin{array}{ll}
\text { color } & r \text { (pname) } \\
\text { palette note (if available) } & r(p) \\
\text { \#th color } & r(p \# \text { info) }
\end{array}
$$

name of palette or custom space-separated list of colors info on \#th color (if available)

\subsection{Viewing a palette (syntax 1$)$}

To display a single palette, type colorpalette followed by the name of the palette. For example, to view the economist palette, type: 
- colorpalette economist

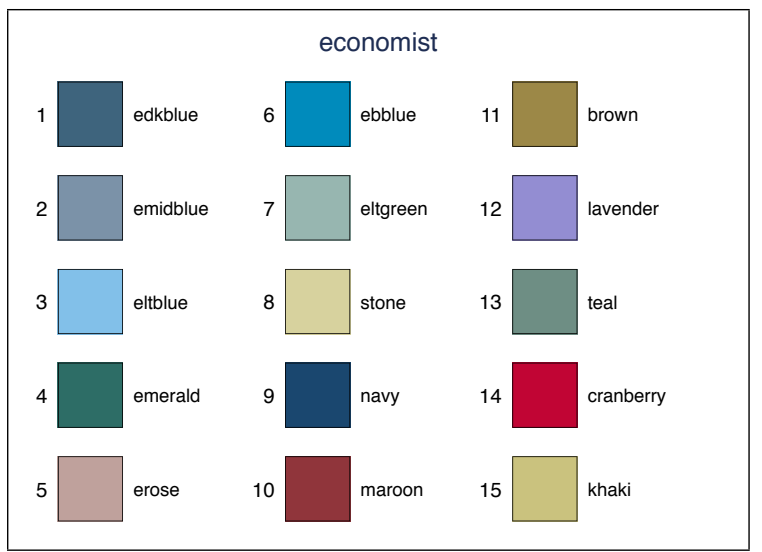

The graph produced by colorpalette displays the colors as well as their names or color codes and, possibly, some additional information. Here is an example of a semantic palette by Lin et al. (2013) with RGB codes and labels:

. colorpalette lin, fruits

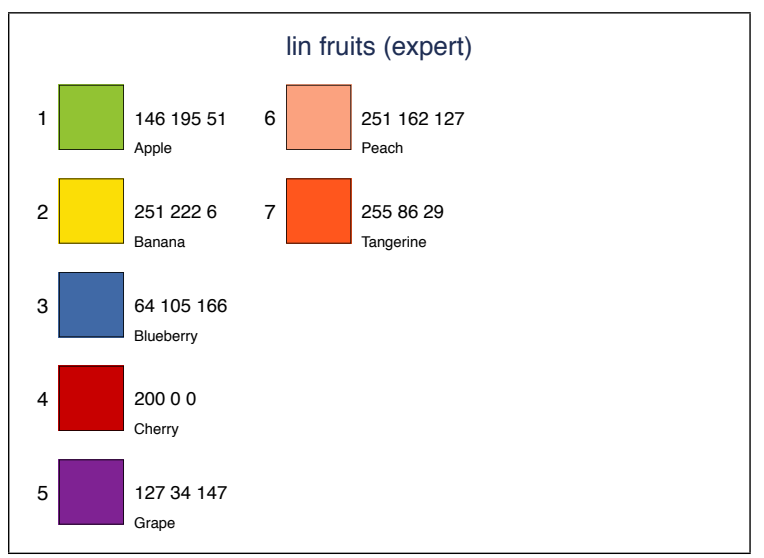

It is also possible to combine colors from multiple palettes. Here is an example that uses the fruits palette, the vegetable palette, and a selection of colors from the food palette by Lin et al. (2013): 

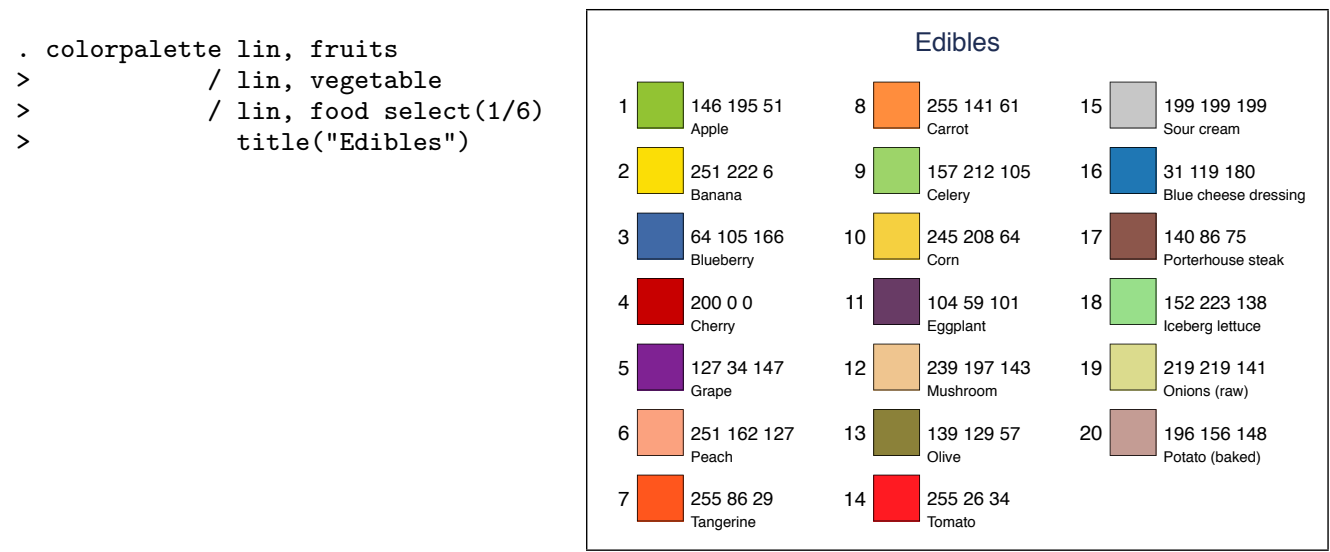

\subsection{Retrieving colors from a palette (syntax 1)}

colorpalette returns the values of the colors in $r()$ so that they can be used in a subsequent graph command. $r(p)$ will contain a space separated list of all colors, $r(p 1), r(p 2)$, etc. will contain the single colors one by one. Here is an example that selects four colors from ColorBrewer's Set1 palette and uses them in a line plot (option nograph is specified to prevent colorpalette from displaying the palette):

- sysuse uslifeexp, clear

(U.S. life expectancy, 1900-1999)

. lab var le_wfemale "white females"

- lab var le_wmale "white males"

. lab var le_bfemale "black females"

- lab var le_bmale "black males"

- colorpalette Set1, $\operatorname{select}(1 / 35)$

$>$ nograph

- line le_wfemale le_wmale

$>\quad$ le_bfemale le_bmale year

$>\quad, \operatorname{lcolor}\left({ }^{\prime} r(p)^{\prime}\right)$ lwidth $(* 2 \ldots)$

$>\quad y t i t l e($ Life expectancy)

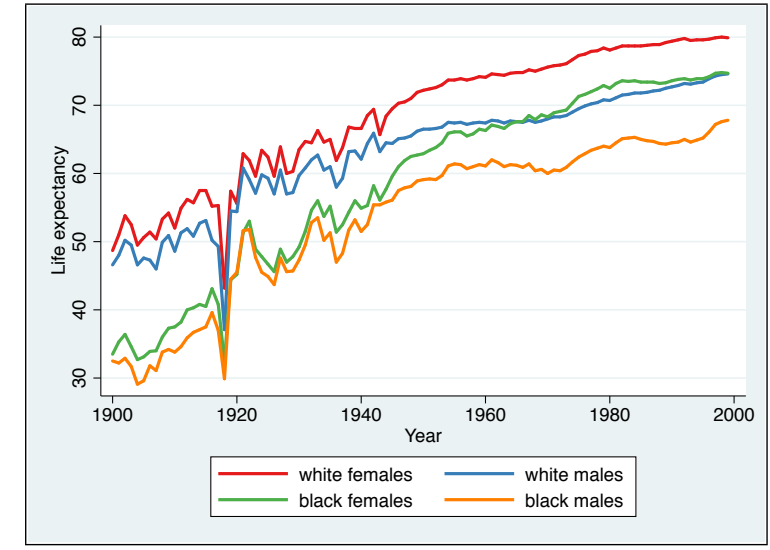

Macro expansion notation ' $r(p)$ ' instructs Stata to paste the contents of $r(p)$ at the specified position within the command. Note that many commands, including most graph commands, clear $\mathrm{r}()$. That is, if you want to use the same colors in multiple graphs without having to call colorpalette repeatedly, copy the colors to a local or global macro ([P] macro). For example, typing 
would copy the list of colors to local macro mycolor. You could then use the colors in subsequent graph commands by typing 'mycolors'.

An alternative is to use the grstyle command to change the default colors used in Stata graphs; grstyle calls colorpalette internally (see Jann 2018).

\subsection{Viewing multiple palettes (syntax 2)}

To display an overview of multiple palettes in a single graph, type colorpalette, a colon, and a list of palettes separated by forward slashes. The following example displays some of the categorical palettes from ColorBrewer (Brewer 2016; Brewer et al. 2003):

- colorpalette, lcolor(black):

$>$ Accent / Dark2 / Set1 / Set3

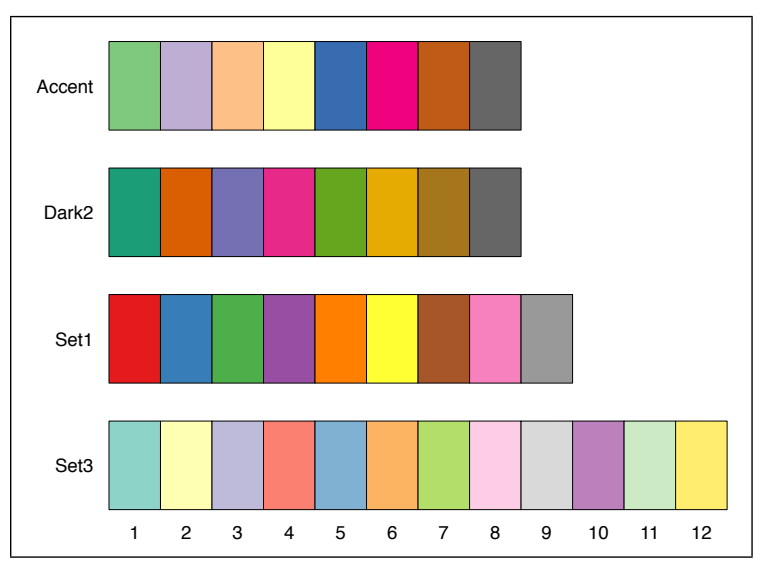

Option lcolor (black) has been specified to draw black lines around the color fields. Separate options can be specified for each palette. Here is an example that displays several default schemes of the HCL color generator. The example also illustrates the effect of the vertical option and the use of $n()$ to determine the number of colors:
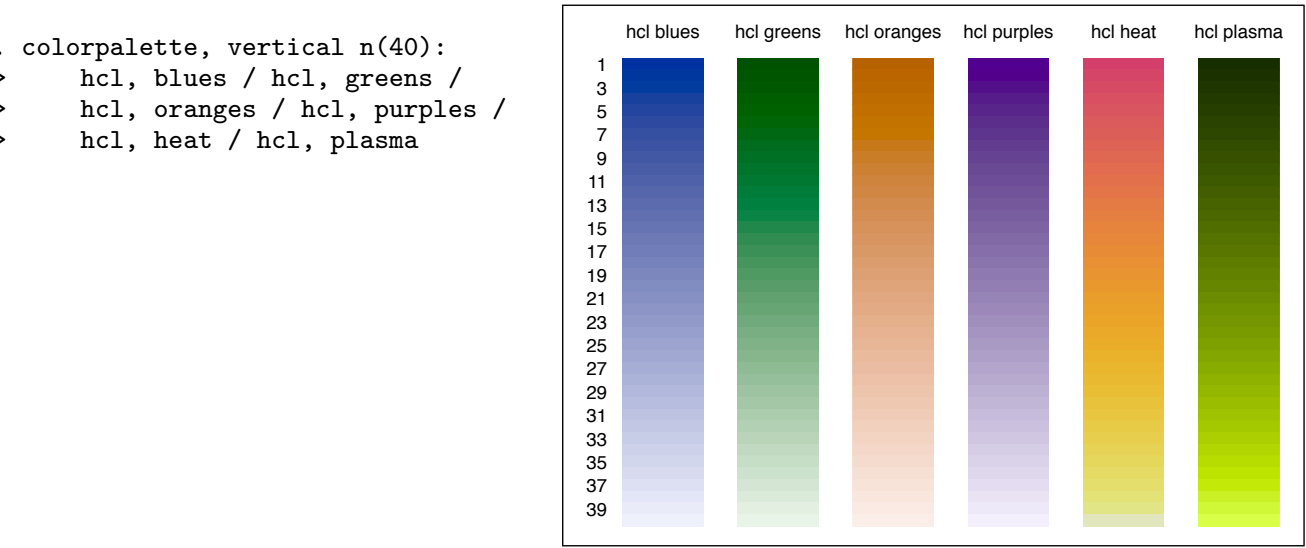


\subsection{Specifying a custom list of colors}

Instead of using a named palette you can provide a custom palette by specifying a list of colorstyles (named colors, RBG values, CMYK values, or HSV values; see [G] colorstyle). Here is an example displaying some of Stata's named colors:
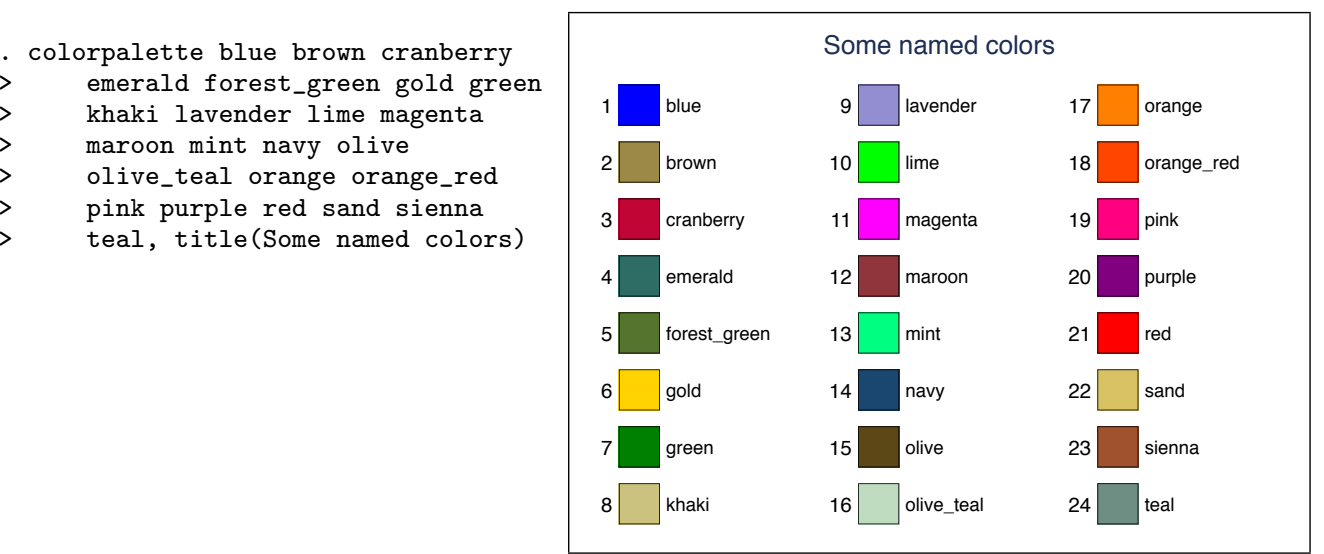

In addition to the color specifications documented in [G] colorstyle, you can specify colors using HCL codes (type "hcl $h \quad c \quad l$ ", where $h, c$, and $l$ are the values for hue, chroma, and luminance) or hex triplets. The specified colors will be translated to RGB. Here is an example displaying (approximate) Federal Standard 595 Safety Colors, using the hex codes found at www.w3schools.com/colors/colors_fs595.asp:
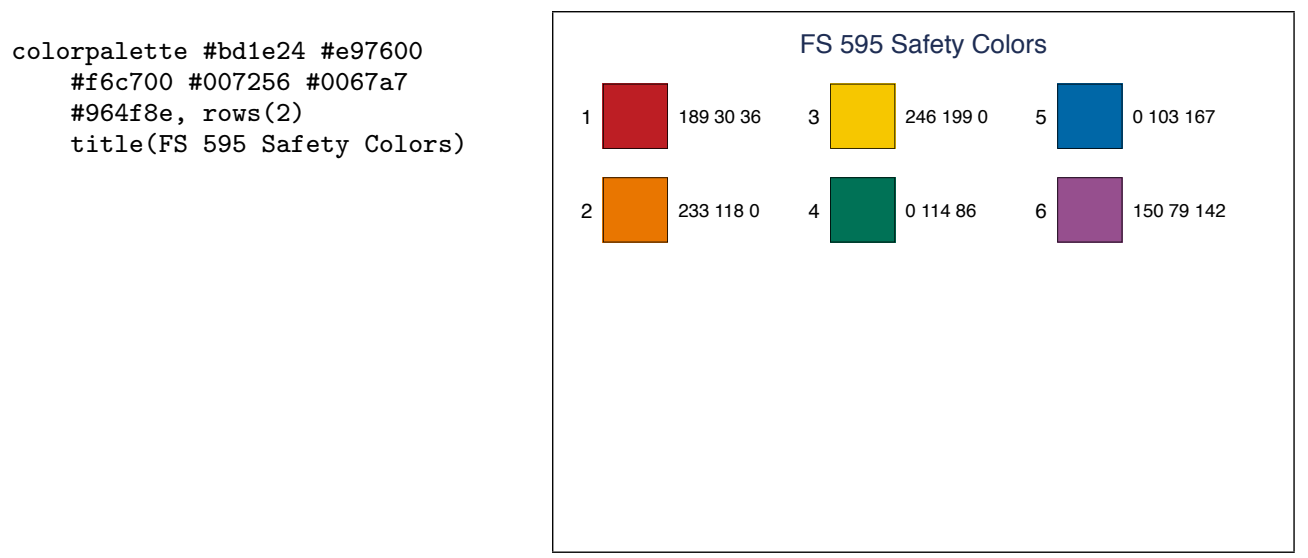

\subsection{Creating colors over a range of intensities or opacity levels}

The intensity() and opacity() options can be used to apply intensity adjustment or assign opacity levels to the selected colors. Both options support number lists as 
argument (see [U] 11.1.8 numlist). If the list of specified numbers is longer than the number of colors in the palette, the list of colors will be recycled. This allows creating colors over a range of intensities or opacity levels, as in the following example:

- colorpalette cranberry,

$>\quad$ intensity $(0.1(.05) 1)$

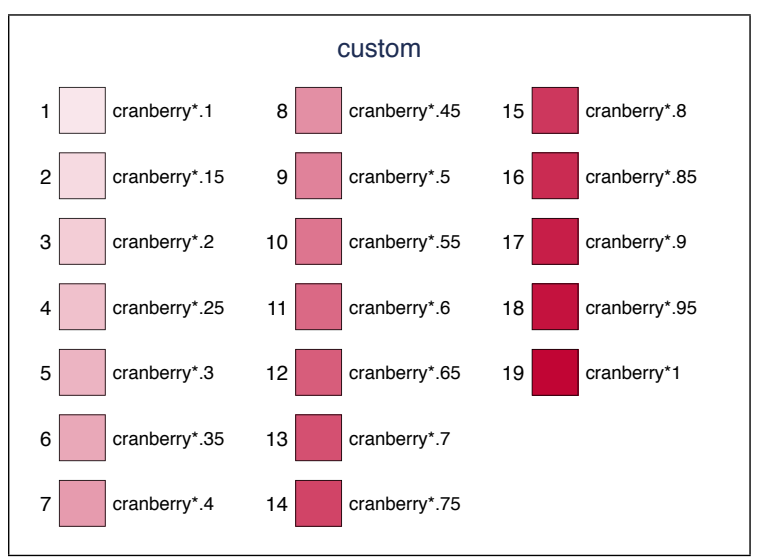

\subsection{Custom palettes}

If you want to create an own named color palette, you can define a program called colorpalette_myname, where myname is the name of your palette. Your program should return the color definitions (e.g., RGB values or hex codes) as a comma-separated list in local macro P. In addition you may provide a comma-separated list of descriptions in local macro I.

After defining the program, the new palette is available to colorpalette like any other palette. Here is an example providing a palette called bootstrap3 containing semantic colors used for buttons in Bootstrap v3.3 (getbootstrap.com/docs/3.3):

- program colorpalette_bootstrap3

1. C_local P \#337ab7,\#5cb85c,

\#5bc0de,\#f0ad4e,\#d9534f

2. c_local I primary, success,

$>\quad$ info, warning, danger

3. end

- colorpalette bootstrap3

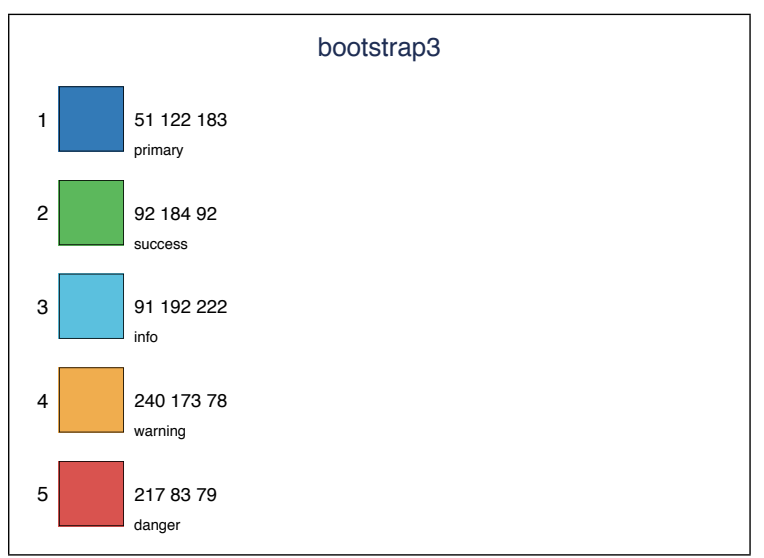


More complicated definitions of palettes that take account of specific options are also possible. See the palette definitions in colorpalette. ado for examples.

If you intend to use the new palette in different analyses, you can store the program in an ado-file instead of including it in each of the do-files. If myname is the name of your palette, the program should be stored in file colorpalette_myname.ado in the working directory or somewhere along Stata's ado path (see [P] sysdir).

\section{Predefined color palettes}

This section provides an overview of the named palettes implemented in colorpalette. There are three types of palettes: Palettes providing the colors used for plots 1 to 15 in official Stata's graph schemes, palettes providing colors found in user-contributed schemes, and collections containing sets of palettes found in the literature.

\subsection{Stata palettes}

The Stata palettes are named after the schemes in which the colors are used. The palettes are:

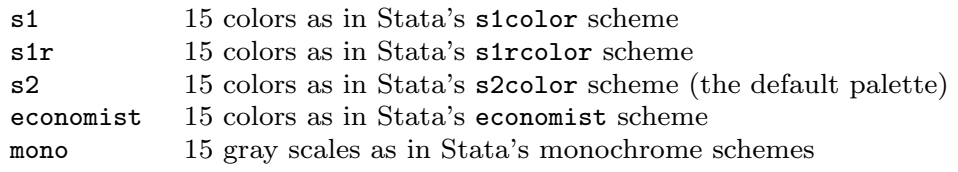

Palette s2 is the default used by colorpalette if no palette is specified. The left panel in Figure 1 displays an overview of the palettes.

\subsection{User-contributed palettes}

Stata users have contributed various scheme files in which alternative sets of colors are used, typically available from the Stata Journal site or from the SSC Archive. The following palettes have been constructed after some of these contributions.

\footnotetext{
cblind 9 colorblind-friendly colors suggested by Okabe and Ito (2002), including an additional gray as suggested at www.cookbook-r.com. The same colors are also used (in different order and using gs10 for gray) in the plotplainblind and plottigblind schemes by Bischof (2017b).

plottig 15 colors used for plots 1 to 15 in the plottig scheme by Bischof (2017b). Most of these colors are the same as the colors produced by the hue color generator with default options (see below), although in different order.

5386 colors used for plots 1 to 6 and 7 colors used for background, labels, axes, and confidence areas in the 538 scheme by Bischof (2017a). The palette replicates colors used at fivethirtyeight.com.

mrc 7 colors used for plots 1 to 7 in the mrc scheme by Morris (2013). These are colors according to guidelines by the UK Medical Research Council.
} 

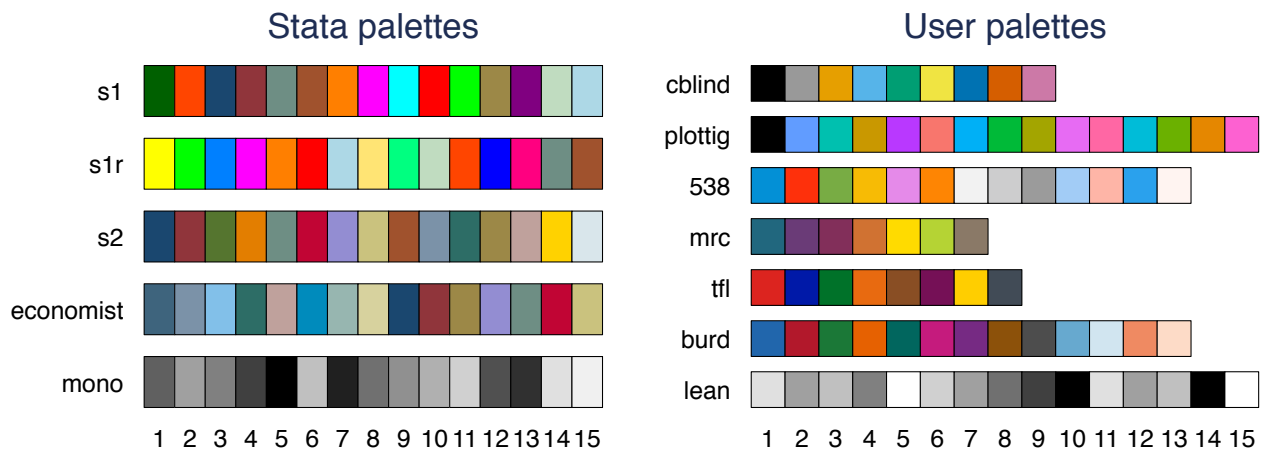

Figure 1: Stata palettes and user-contributed palettes

\begin{abstract}
tfl 8 colors used for plots 1 to 8 in the tfl scheme by Morris (2015). The palette replicates Transport for London's corporate colors.

burd 9 colors used for plots 1 to 9 and 4 colors used for confidence areas in the burd scheme by Briatte (2013). The first 9 colors are a selection of colors from various ColorBrewer schemes.

lean 15 gray scales used for areas in plots 1 to 15 in schemes lean1 and lean2 by Juul (2003).
\end{abstract}

The right panel in Figure 1 displays an overview of these palettes.

\title{
3.3 Collections
}

\section{ColorBrewer}

ColorBrewer is a set of color schemes developed by Brewer et al. (2003; also see Brewer 2016). For more information on ColorBrewer also see colorbrewer2.org. ${ }^{1}$ The syntax for the ColorBrewer palettes is

scheme [, cmyk palette_options ]

where palette_options are general palette options as described above, cmyk requests the CMYK variant of the colors instead of the RGB variant, and scheme is one of the following:

1. The colors are licensed under Apache License Version 2.0; see the copyright notes at www.personal.psu.edu/cab38/ColorBrewer/ColorBrewer_updates.html. The RGB values for the implementation of the colors in colorpalette have been taken from the Excel spreadsheet provided at www.personal.psu.edu/cab38/ColorBrewer/ColorBrewer_RGB.html. The CMYK values have been taken from file cb.csv provided at github.com/axismaps/colorbrewer. ColorBrewer palettes for Stata have also been provided by Gomez (2015) and by Buchanan (2015). 
Qualitative schemes

Accent 8 accented colors for qualitative data Paired 12 paired colors for qualitative data

Pastel2 8 pastel colors for qualitative data

Set2 8 colors for qualitative data

Dark2 8 dark colors for qualitative data

Pastel1 9 pastel colors for qualitative data

Set1 9 colors for qualitative data

Set3 12 colors for qualitative data

Single-hue sequential schemes (3-9 colors)

Blues light blue to blue

Greys light gray to gray

Purples light purple to purple

Multi-hue sequential schemes (3-9 colors)

$\mathrm{BuGn} \quad$ light blue to green

$\mathrm{GnBu} \quad$ light green to blue

$\mathrm{PuBu} \quad$ light purple to blue

PuRd light purple to red

YIGn light yellow to green

Y1OrBr light yellow over orange to brown

Greens light green to green

Oranges light orange to orange

Reds light red to red

$\mathrm{BuPu} \quad$ light blue to purple

OrRd light orange to red

PuBuGn light purple over blue to green

$\mathrm{RdPu} \quad$ light red to purple

YIGnBu light yellow over green to blue

Y1OrRd light yellow over orange to red

Diverging schemes (3-11 colors)

$\begin{array}{llll}\text { BrBG } & \text { brown to green, light gray mid } & \text { PiYG } & \text { pink to green, light gray mid } \\ \text { PRGn } & \text { purple to green, light gray mid } & \text { PuOr } & \text { purple to orange, light gray mid } \\ \text { RdBu } & \text { red to blue, light gray mid } & \text { RdGy } & \text { red to gray, white mid } \\ \text { RdYlBu } & \text { red to blue, yellow mid } & \text { RdYlGn } & \text { red to green, yellow mid } \\ \text { Spectral } & \text { red - orange - yellow - green - blue } & & \end{array}$

Figure 2 display the schemes (using the maximum number of colors for those schemes that come in different sizes).

\section{Semantic colors by Lin et al.}

The Iin collection provides semantic color schemes suggested by Lin et al. (2013). ${ }^{2}$ The syntax is

lin [, scheme algorithm palette_options ]

where palette_options are general palette options as discussed above and scheme is one of the following:

$\begin{array}{llll}\text { tableau } & 20 \text { categorical colors; the default } & \\ \text { carcolor } & 6 \text { car colors } & \text { food } & 7 \text { food colors } \\ \text { features } & 5 \text { feature colors } & \text { activities } & 5 \text { activity colors } \\ \text { fruits } & 7 \text { fruit colors } & \text { vegetables } & 7 \text { vegetable colors } \\ \text { drinks } & 7 \text { drinks colors } & \text { brands } & 7 \text { brands colors }\end{array}$

Option algorithm requests algorithm selected colors. The default is to return the colors selected by Turkers (in case of carcolor, food, features, activities) or by the expert (in case of fruits, vegetables, drinks, brands). Option algorithm has no effect for tableau. Figure 3 display the schemes.

2. The values of the semantic colors have been taken from the source code of the brewscheme package by Buchanan (2015) (brewextra.ado, version 1.0.0, 21 March 2016); the values of the tableau palette have been taken from code provided by Lin et al. at github.com/StanfordHCI/semanticcolors. 
Qualitative

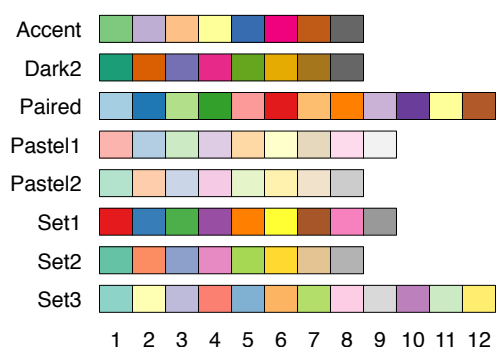

Diverging

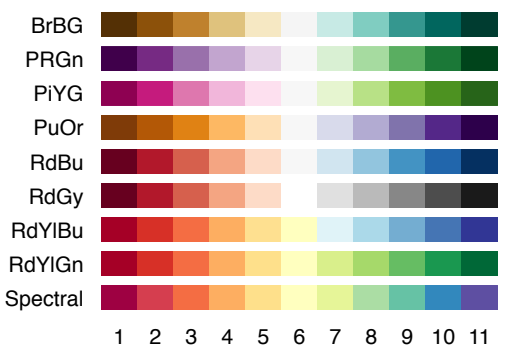

\section{Sequential (single hue)}

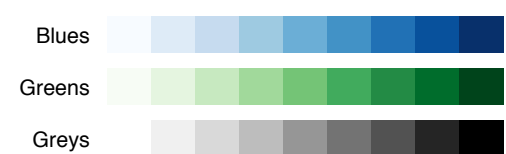

Oranges

Purples

Reds

$\begin{array}{lllllllll}1 & 2 & 3 & 4 & 5 & 6 & 7 & 8 & 9\end{array}$

Sequential (multi-hue)

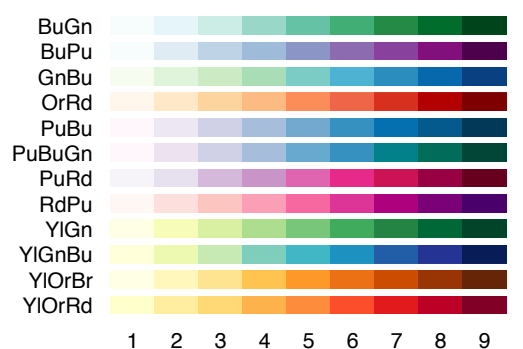

Figure 2: ColorBrewer schemes
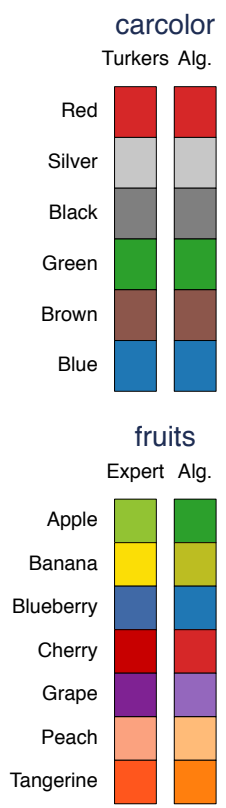
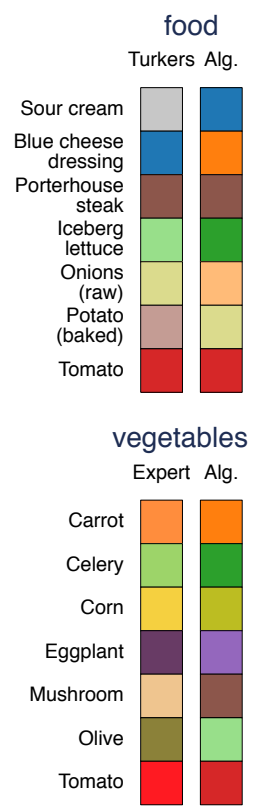
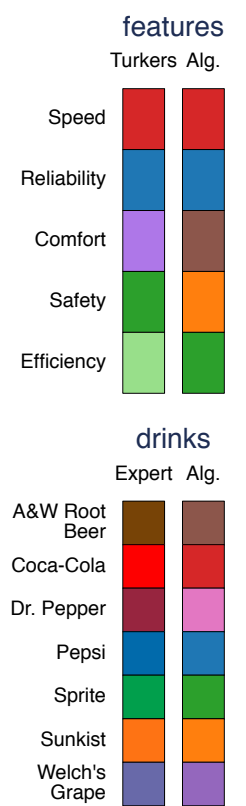
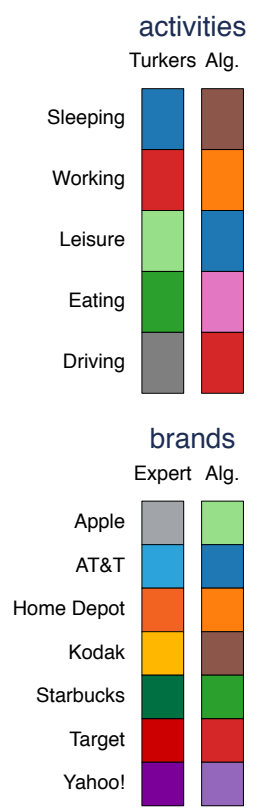

Figure 3: Semantic color schemes by Lin et al. (2013) 


\section{Color schemes by Paul Tol}

The ptol collection provides color schemes as suggested by Tol (2012). The syntax is

ptol [, scheme palette_options ]

where palette_options are general palette options as discussed above and scheme is one of the following (displayed for for different numbers of colors in the upper left panel in Figure 4).

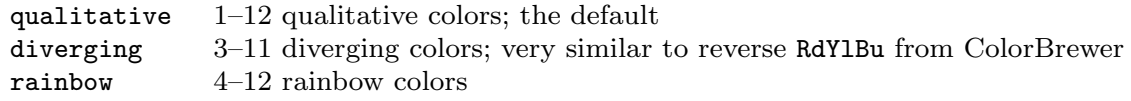

\section{D3.js}

The d3 collection provides color schemes from d3js.org, using the color values found at github.com/d3/d3-scale/blob/master/README.md\#category-scales. The syntax is

d3 [, scheme palette_options ]

where palette_options are general palette options as discussed above and scheme is one of the following (displayed in the upper right panel in Figure 4).

1010 categorical colors; the default; same as the first 10 colors in the tableau scheme of the lin collection

2020 categorical colors in pairs; same colors as in the tableau scheme of the lin collection, but in different order

$20 \mathrm{~b} \quad 20$ categorical colors in groups of four

20c 20 categorical colors in groups of four

\section{Colors schemes from spmap}

The spmap collection provides color schemes from the spmap package by Pisati (2007). The implementation is based on code from spmap_color.ado (version 1.3.0, 13 March 2017). The syntax is:

spmap [, scheme palette_options ]

where palette_options are general palette options as discussed above and scheme is one of the following (displayed for $\mathrm{n}(16)$ in the lower left panel in Figure 4).

\begin{tabular}{|c|c|c|c|}
\hline blues & $\begin{array}{l}\text { light blue to blue (2-99 colors); } \\
\text { the default }\end{array}$ & greens & $\begin{array}{l}\text { light green to green }(2-99 \\
\text { colors) }\end{array}$ \\
\hline & light gray to black (2-99 colors) & reds & light red to red (2-99 colors \\
\hline & $2-99$ rainbow colors & heat & $2-16$ heat colors \\
\hline terrain & $2-16$ terrain colors & topological & $2-16$ topological colors \\
\hline
\end{tabular}



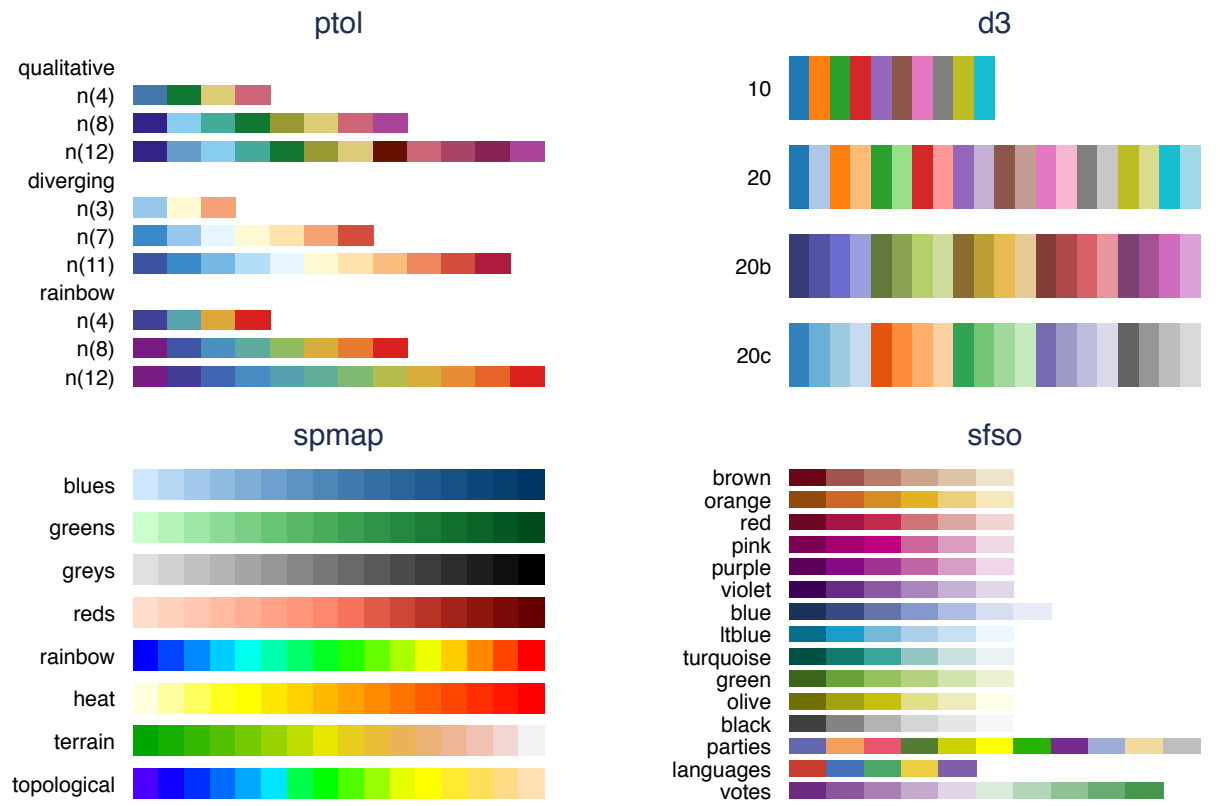

Figure 4: Various palettes

\section{Swiss Federal Statistical Office colors}

The sf so collection provides color schemes by the Swiss Federal Statistical Office (using hex and CMYK codes found in Bundesamt für Statistik 2017). The syntax is

sfso [, scheme cmyk palette_options ]

where palette_options are general palette options as discussed above and scheme is one of the following (displayed in the lower right panel in Figure 4).

Sequential schemes

\begin{tabular}{|c|c|c|c|}
\hline brown & dark brown to light brown & orange & dark orange to light orange \\
\hline red & dark red to light red & pink & dark pink to light pink \\
\hline purple & dark purple to light purple & violet & dark violet to light violet \\
\hline blue & dark blue to light blue; the default & ltblue & lighter version of blue \\
\hline turquoise & dark turquoise to light turquoise & green & dark green to light green \\
\hline olive & dark olive to light olive & black & dark gray to light gray \\
\hline & & res & nяия \\
\hline $\begin{array}{l}\text { arties } \\
\text { otes }\end{array}$ & $\begin{array}{l}\text { colors used for Swiss parties } \\
\text { colors used for results from votes }\end{array}$ & languages & colors used for languages \\
\hline
\end{tabular}

Option cmyk requests the CMYK variant of a scheme. The default is to use the RGB variant. 


\section{Color generators}

\subsection{Evenly spaced HCL hues}

The hue palette implements an algorithm that generates HCL colors with evenly spaced hues. The palette has been modeled after function hue_pal() from R's scales package by Hadley Wickham (see github.com/hadley/scales). This is the default color scheme used by R's ggplot2 for categorical data (see ggplot2.tidyverse.org/reference/ scale_hue.html). The hue palette with default options produces the same colors as the intense scheme of the hcl color generator (see below). The syntax of the hue color generator is

hue [, hue_options palette_options ]

where palette_options are general palette options as discussed above and hue_options are:

hue $\left(h_{1} h_{2}\right)$ sets the range of hues on the 360 degree color wheel. The default is hue (15 375 ). If the difference between start and end is a multiple of 360 , end will be reduced by $360 / n$, where $n$ is the number of requested colors (so that the space between the last and the first color is the same as between the other colors).

chroma(c) sets the colorfulness (color intensity), with $c \geq 0$. The default is chroma (100).

luminance $(l)$ sets the brightness (amount of gray), with $l \in[0,100]$. The default is luminance (65).

direction(\#) determines the direction to travel around the color wheel. direction(1), the default, travels clockwise; direction $(-1)$ travels counter-clockwise.

The following graph illustrates how the colors change depending on option $\mathrm{n}()$ :
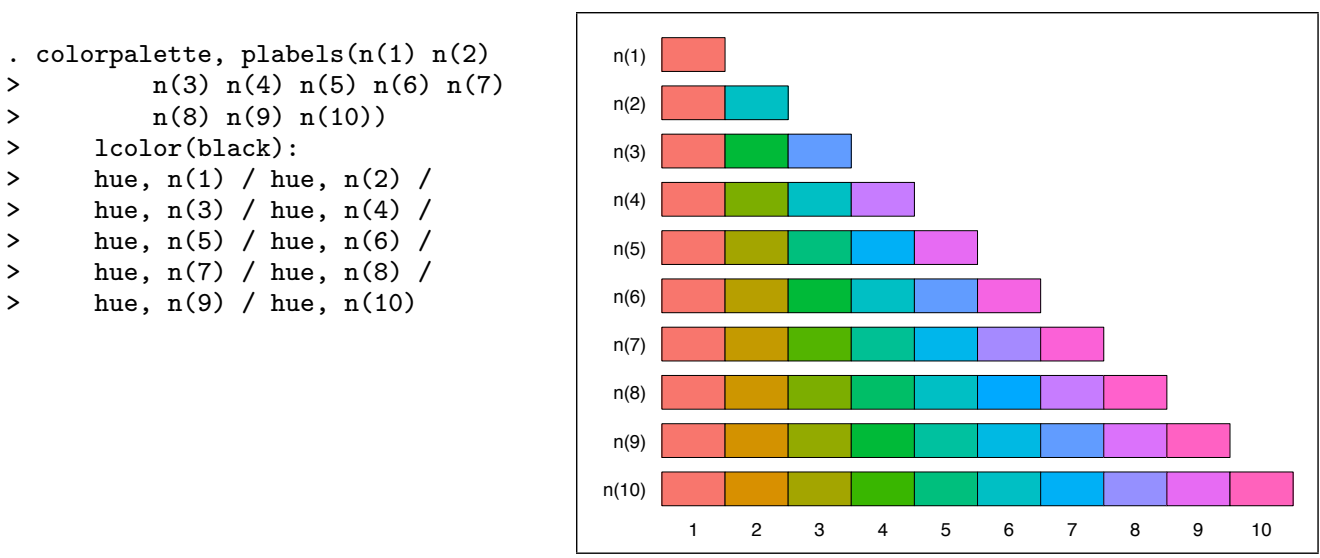


\subsection{HCL color generator}

The hcl palette is a HCL color generator (Hue-Chroma-Luminance). The implementation is based on R's colorspace package by Ihaka et al. (2016); also see Zeileis et al. (2009) and hclwizard.org.

Let $h_{1}$ and $h_{2}$ be two hues on the 360 degree color wheel, $c_{1}$ and $c_{2}$ two chroma levels, $l_{1}$ and $l_{2}$ two luminance levels, $p_{1}$ and $p_{2}$ two power parameters, and $i$ an index from 1 to $n$, where $n$ is the number of requested colors. The HCL colors are then generated according to the following formulas.

Qualitative schemes:

$$
H=h_{1}+j\left(h_{2}-h_{1}\right), \quad C=c_{1}, \quad L=l_{1}, \quad j=\frac{i-1}{n-1}
$$

Sequential schemes:

$$
H=h_{2}-j\left(h_{2}-h_{1}\right), \quad C=c_{2}-j^{p_{1}}\left(c_{2}-c_{1}\right), \quad L=l_{2}-j^{p_{2}}\left(l_{2}-l_{1}\right), \quad j=\frac{n-i}{n-1}
$$

Diverging schemes:

$$
H=\left\{\begin{array}{ll}
h_{1} & \text { if } j>0 \\
h_{2} & \text { else }
\end{array}, \quad C=|j|^{p_{1}} c_{1}, \quad L=l_{2}-|j|^{p_{2}}\left(l_{2}-l_{1}\right), \quad j=\frac{n-2 j+1}{n-1}\right.
$$

The syntax of the hcl color generator is

hcl [, scheme hcl_options palette_options ]

\begin{tabular}{|c|c|c|c|c|c|c|c|c|c|c|c|c|c|c|c|c|c|}
\hline \multirow[b]{2}{*}{$\begin{array}{l}\text { Qualitative } \\
\text { qualitative }\end{array}$} & $\underline{h_{1}}$ & $h_{2}$ & $c_{1}$ & $c_{2}$ & $l_{1}$ & $l_{2}$ & $p_{1}$ & $p_{2}$ & \multirow[b]{2}{*}{ intense } & \multicolumn{2}{|c|}{$\begin{array}{ll}h_{1} & h_{2} \\
\end{array}$} & & $c_{2}$ & $l_{1}$ & $l_{2}$ & $p_{1}$ & $p_{2}$ \\
\hline & 15 & $h^{*}$ & 60 & - & 70 & - & - & - & & 15 & $h^{*}$ & 100 & - & 65 & 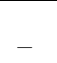 & - & - \\
\hline$\frac{1}{\text { dark }}$ & 15 & $h^{*}$ & 80 & - & 60 & - & - & - & light & 15 & $h^{*}$ & 50 & - & 80 & - & - & - \\
\hline & 15 & $h^{*}$ & 35 & - & 85 & - & - & - & \multicolumn{9}{|c|}{ with $h^{*}=h_{1}+360(n-1) / r$} \\
\hline \multicolumn{18}{|l|}{ Sequential } \\
\hline sequential & 260 & $h_{1}$ & 80 & 10 & 25 & 95 & 1 & $p_{1}$ & b7 & 260 & $h_{1}$ & 80 & 10 & 25 & 95 & 1 & $p_{1}$ \\
\hline greens & 145 & 125 & 80 & 10 & 25 & 95 & 1 & & & 0 & $h_{1}$ & 0 & 0 & 15 & 95 & 1 & $p_{1}$ \\
\hline orange & 40 & $h_{1}$ & 100 & 10 & 50 & 95 & 1 & & & 280 & $h_{1}$ & 70 & 10 & 20 & 95 & 1 & $p_{1}$ \\
\hline reds & 10 & 20 & 80 & 10 & 25 & 95 & 1 & $p_{1}$ & eat & 0 & 90 & 100 & 30 & 50 & 90 & .2 & 1 \\
\hline & 0 & 90 & 80 & 30 & 30 & 90 & .2 & 2 & 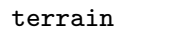 & 130 & & 80 & 0 & 60 & 95 & .1 & 1 \\
\hline & & 30 & 65 & 0 & 45 & 90 & .5 & 1.5 & & 300 & & 35 & 95 & 15 & 90 & .8 & 1.2 \\
\hline plasma & & $h_{1}$ & 60 & 100 & & 95 & 2 & .9 & & 0 & -100 & 80 & 40 & 40 & 75 & 1 & 1 \\
\hline \multicolumn{18}{|l|}{ Diverging } \\
\hline diverging & & 0 & 80 & - & 30 & 95 & 1 & $p_{1}$ & & 260 & & 80 & - & 30 & 95 & 1 & $p_{1}$ \\
\hline & 260 & 0 & 100 & - & 50 & & 1 & $p$ & blu & 180 & 330 & 60 & - & 75 & 95 & 1 & $p_{1}$ \\
\hline & 130 & 45 & 100 & - & 70 & & 1 & $p$ & brownore & 55 & 160 & 60 & - & 35 & 95 & 1 & \\
\hline pinkgreen & 340 & 128 & 90 & - & 35 & 95 & 1 & $p_{1}$ & purplegreen & 300 & 128 & 60 & - & 30 & 95 & 1 & $p_{1}$ \\
\hline
\end{tabular}

where palette_options are general palette options as discussed above and scheme selects the type of scheme and the default parameters according to the following overview: 
hcl_options are:

hue $\left(h_{1}\left[h_{2}\right]\right)$ overwrites the default values for $h_{1}$ and $h_{2}$ that determine the range of hues on the 360 degree color wheel.

chroma $\left(c_{1}\left[c_{2}\right]\right)$ overwrites the default values for $c_{1}$ and $c_{2}, c_{i} \geq 0$, that determine the colorfulness (color intensity).

Iuminance $\left(l_{1}\left[l_{2}\right]\right)$ overwrites the default values for $l_{1}$ and $l_{2}, l_{i} \in[0,100]$, that determine the brightness (amount of gray).

power $\left(p_{1}\left[p_{2}\right]\right)$ overwrites the default values for $p_{1}$ and $p_{2}, p_{i}>0$, that determine the shape of the transition between chroma and luminance levels. For linear transitions, set $p_{i}=1 ; p_{i}>1$ makes the transition faster, $p_{i}<1$ makes the transition slower.

The left panel of Figure 5 displays the predefined HCL schemes with default parameters for $n=15$.

\subsection{HSV color generator}

The hsv palette is a HSV color generator (Hue-Saturation-Value). The implementation is partially based on R's grDevices package (which is part of the R core) and partially on colorspace by Ihaka et al. (2016).

Let $h_{1}$ and $h_{2}$ be two hues on the 360 degree color wheel, $s_{1}$ and $s_{2}$ two saturation levels, $v_{1}$ and $v_{2}$ two value levels, $p_{1}$ and $p_{2}$ two power parameters, and $i$ an index from 1 to $n$, where $n$ is the number of requested colors. The HSV colors are then generated according to the following formulas.

Qualitative schemes:

$$
H=h_{1}+j\left(h_{2}-h_{1}\right), \quad S=s_{1}, \quad V=v_{1}, \quad j=\frac{i-1}{n-1}
$$

Sequential schemes:

$$
H=h_{2}-j\left(h_{2}-h_{1}\right), \quad S=s_{2}-j^{p_{1}}\left(s_{2}-s_{1}\right), \quad V=v_{2}-j^{p_{2}}\left(v_{2}-v_{1}\right), \quad j=\frac{n-i}{n-1}
$$

Diverging schemes:

$$
H=\left\{\begin{array}{ll}
h_{1} & \text { if } j>0 \\
h_{2} & \text { else }
\end{array}, \quad S=|j|^{p_{1}} s_{1}, \quad V=v_{2}-|j|^{p_{2}}\left(v_{2}-v_{1}\right), \quad j=\frac{n-2 j+1}{n-1}\right.
$$

The syntax of the hsv color generator is

hsv [, scheme hsv_options palette_options ]

where palette_options are general palette options as discussed above and scheme selects the type of scheme and the default parameters according to the following overview: 


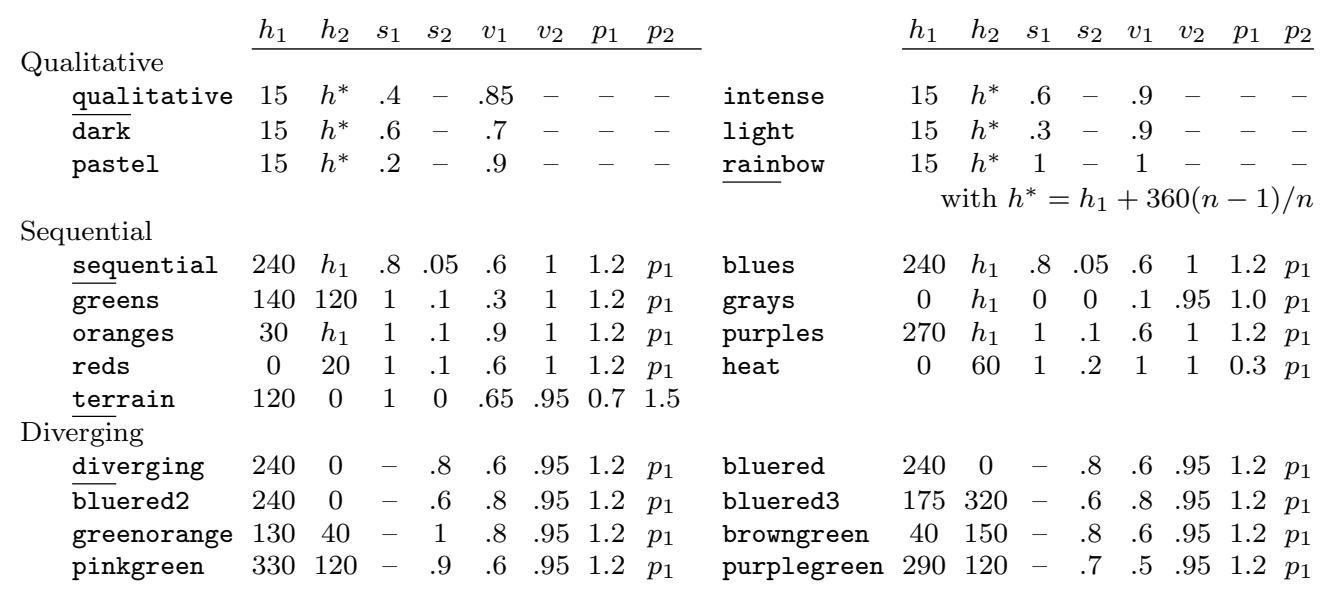

hsv_options are:

hue $\left(h_{1}\left[h_{2}\right]\right)$ overwrites the default values for $h_{1}$ and $h_{2}$ that determine the range of hues on the 360 degree color wheel.

saturation $\left(s_{1}\left[s_{2}\right]\right.$ ) overwrites the default values for $s_{1}$ and $s_{2}, s_{i} \in[0,1]$, that determine the colorfulness (color intensity).

$\operatorname{value}\left(v_{1}\left[v_{2}\right]\right)$ overwrites the default values for $v_{1}$ and $v_{2}, v_{i} \in[0,1]$, that determine the brightness (amount of gray).

power $\left(p_{1}\left[p_{2}\right]\right)$ overwrites the default values for $p_{1}$ and $p_{2}, p_{i}>0$, that determine the shape of the transition between chroma and luminance levels. For linear transitions, set $p_{i}=1 ; p_{i}>1$ makes the transition faster, $p_{i}<1$ makes the transition slower.

The right panel of Figure 5 displays the predefined HSV schemes with default parameters for $n=15$.

\section{Appendix: Symbol palettes and line pattern palettes}

The palettes package also contains commands for symbol palettes and line pattern palettes. Their syntax and basic functionality is similar to the command for color palettes; see help symbolpalette and help linepalette. Figure 6 display an overview of the available named palettes.

\section{References}

Bischof, D. 2017a. G538SCHEMES: module to provide graphics schemes for http://fivethirtyeight.com. Statistical Software Components S458404, Boston College Department of Economics. Available from https://ideas.repec.org/c/boc/bocode/s458404.html.

- 2017b. New graphic schemes for Stata: plotplain and plottig. The Stata Journal 
Qualitative HCL

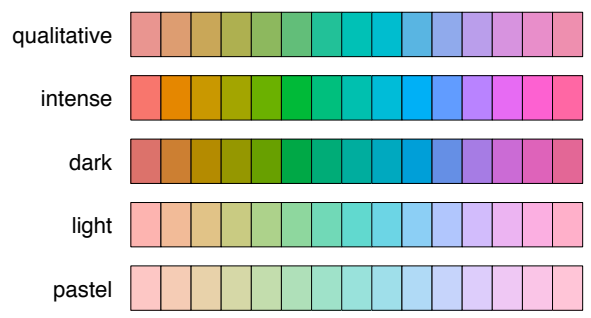

Sequential HCL (single hue)

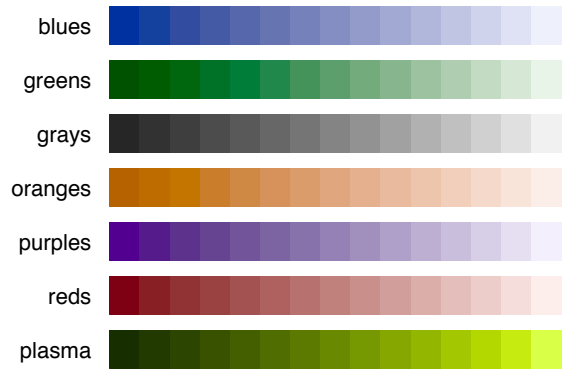

Sequential HCL (multi-hue)

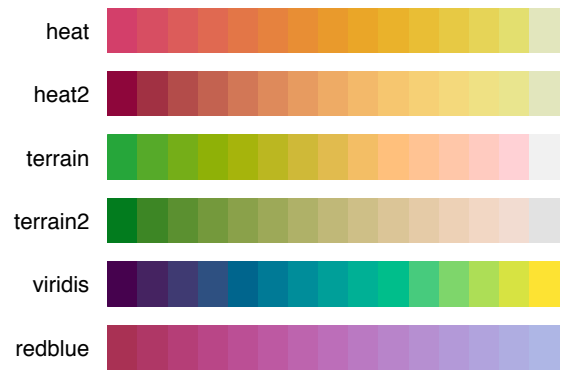

Diverging $\mathrm{HCL}$

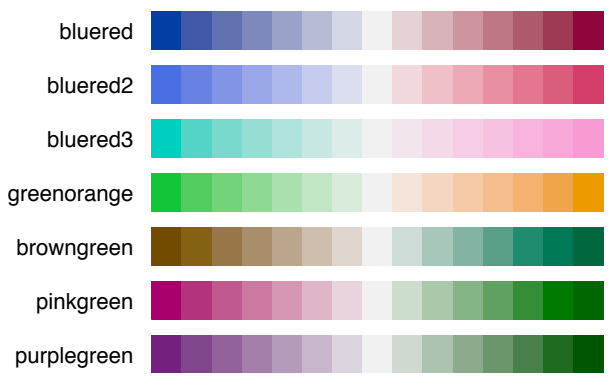

Qualitative HSV (single hue)

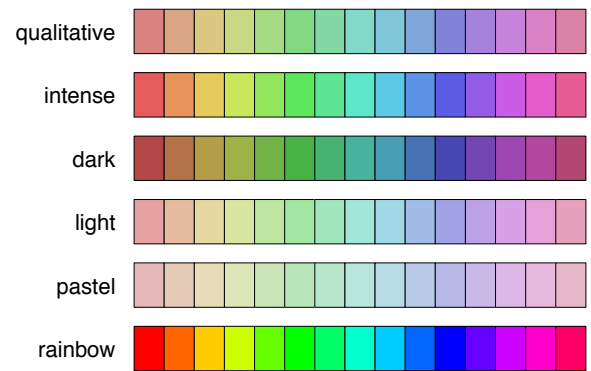

Sequential HSV

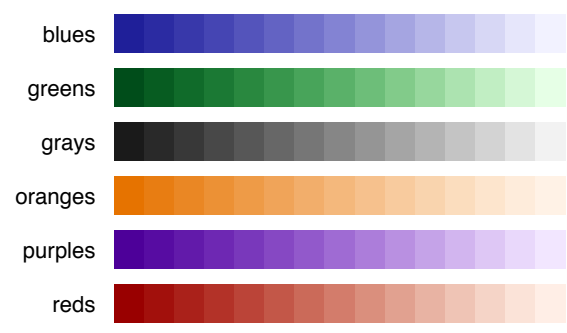

Sequential HSV (multi-hue) heat terrain

\section{Diverging HSV}

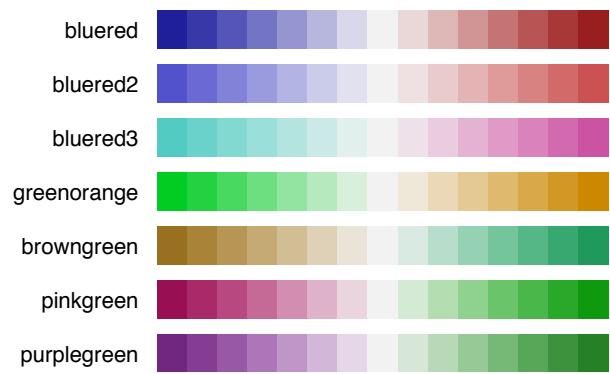

Figure 5: HCL and HCV color schemes 

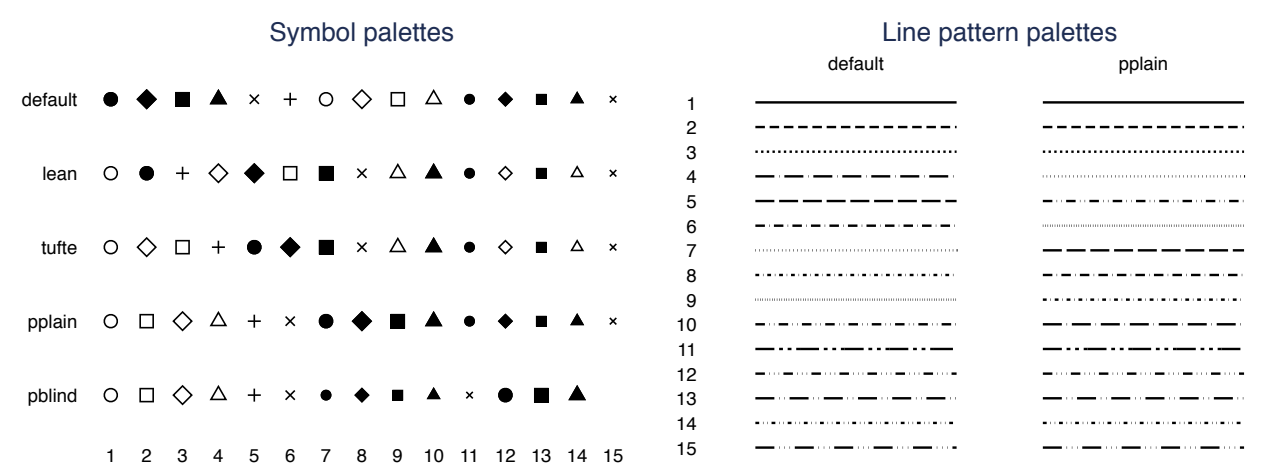

Figure 6: Symbol palettes and line pattern palettes

17(3): 748-759.

Brewer, C. A. 2016. Designing Better Maps. A Guide for GIS Users. 2nd ed. Redlands, CA: Esri Press.

Brewer, C. A., G. W. Hatchard, and M. A. Harrower. 2003. ColorBrewer in Print: A Catalog of Color Schemes for Maps. Cartography and Geographic Information Science 30(1): 5-32.

Briatte, F. 2013. SCHEME-BURD: Stata module to provide a ColorBrewer-inspired graphics scheme with qualitative and blue-to-red diverging colors. Statistical Software Components S457623, Boston College Department of Economics. Available from https://ideas.repec.org/c/boc/bocode/s457623.html.

Buchanan, B. 2015. BREWSCHEME: Stata module for generating customized graph scheme files. Statistical Software Components S458050, Boston College Department of Economics. Available from https://ideas.repec.org/c/boc/bocode/s458050.html.

Bundesamt für Statistik. 2017. Layoutrichtlinien. Gestaltungs und Redaktionsrichtlinien für Publikationen, Tabellen und grafische Assets. Technical Report Version 1.1.1, Bundesamt für Statistik, Neuchâtel.

Gomez, M. 2015. Stata command to generate color schemes. Available from http://github.com/matthieugomez/stata-colorscheme.

Ihaka, R., P. Murrell, K. Hornik, J. C. Fisher, R. Stauffer, and A. Zeileis. 2016. colorspace: Color Space Manipulation. R package version 1.3-2. Available from http://CRAN.R-project.org/package=colorspace.

Jann, B. 2018. Customizing Stata graphs made even easier. University of Bern Social Sciences Working Papers 30, available from http://ideas.repec.org/p/bss/wpaper/30.html. 
Juul, S. 2003. Lean mainstream schemes for Stata 8 graphics. The Stata Journal 3(3): 295-301.

Lin, S., J. Fortuna, C. Kulkarni, M. Stone, and J. Heer. 2013. Selecting SemanticallyResonant Colors for Data Visualization. Computer Graphics Forum 32(3pt4): 401410 .

Morris, T. 2013. SCHEME-MRC: Stata module to provide graphics scheme for UK Medical Research Council. Statistical Software Components S457703, Boston College Department of Economics. Available from https://ideas.repec.org/c/boc/bocode/s457703.html.

2015. SCHEME-TFL: Stata module to provide graph scheme, based on Transport for London's corporate colour pallette. Statistical Software Components S458103, Boston College Department of Economics. Available from https://ideas.repec.org/c/boc/bocode/s458103.html.

Okabe, M., and K. Ito. 2002. Color Universal Design (CUD). How to make figures and presentations that are friendly to Colorblind people. Available from http://jfly.iam.utokyo.ac.jp/color/.

Pisati, M. 2007. SPMAP: Stata module to visualize spatial data. Available from http://ideas.repec.org/c/boc/bocode/s456812.html.

Tol, P. 2012. Colour Schemes. SRON Technical Note, Doc. no. SRON/EPS/TN/09-002. Available from https://personal.sron.nl/ ${ }^{\sim}$ pault/colourschemes.pdf.

Zeileis, A., K. Hornik, and P. Murrell. 2009. Escaping RGBland: Selecting Colors for Statistical Graphics. Computational Statistics \& Data Analysis 53: 3259-3270.

\section{About the author}

Ben Jann is Professor of Sociology at the University of Bern, Switzerland. His research interests include social-science methodology, statistics, social stratification, and labor market sociology. He is principle investigator of TREE, a large-scale multi-cohort panel study in Switzerland on transitions from education to employment (www.tree.unibe.ch). 